\title{
Consenso colombiano de broncoscopia durante la pandemia y pospandemia por SARS-CoV-2
}

\author{
Bronchoscopy during the COVID-19 pandemic and \\ post-pandemic: Colombian (ASONEUMOCITO/ \\ ACBNI) guideline and expert panel report
}

\begin{abstract}
ISABel Palacios-Ortega, MD ${ }^{1,2}$, Fabio Bolívar-Grimaldos, MD ${ }^{1,3}$, John EduARdo Bastidas, MD ${ }^{1}$, Diana Cano-Rosales, MD ${ }^{1}$, Alejandra Cañas, MD ${ }^{1,4}$, Hugo Caballero, MD ${ }^{1,5}$, Felipe Campo-Campo, MD ${ }^{1,6}$, JAIME ECHEVERRI-FRANCO, MD ${ }^{1,7}$, LILIANA FERnÁNDEZ-TRUJILLO, MD ${ }^{1,8}$, ALEJANDRo LondoÑoVillegas, MD ${ }^{1}$, Carlos Eduardo Matiz, MD, FCCP ${ }^{1}$, José Ezequiel Mier-Osejo, MD ${ }^{1,9}$, Nelson PÁez-Espinel, MD ${ }^{1,10}$, HÉctor EnRIQue Paúl, MD ${ }^{1}$, Jaime SÁnchez Vallejo, MD ${ }^{1,11}$, Franco MontufarANDRADE, MD ${ }^{1,12}$, Oscar Alberto SÁENZ-MORALES, MD ${ }^{1}$
\end{abstract}

\section{Introducción}

La enfermedad por coronavirus 2019 (COVID-19) es el nombre dado a la patología causada por la infección por el coronavirus del síndrome respiratorio agudo severo 2 (SARS-CoV-2) $(1,2)$.

Teniendo en cuenta la actual fase de mitigación, la alta tasa de contagio, así como la exposición a la que se ve expuesto el broncoscopista y demás personal en un procedimiento altamente productor de aerosoles, se hace necesaria la actualización de guías sobre recomendaciones en broncoscopia previamente publicadas, dada la necesidad de reapertura de los servicios ambulatorios y la atención de pacientes hospitalizados por otras patologías.

Con alto nivel de contagio conocido, y el riesgo para el personal de salud, este se considera como un procedimiento contraindicado como método diagnóstico en COVID-19, excepto en condiciones que amenacen la vida y cuyo resultado cambie el manejo médico del paciente (3-7).

La actual guía es un consenso de expertos, miembros del Comité de Broncoscopia de la Asociación Colombiana de Neumología y Cirugía de Tórax. Al momento, la evidencia se encuentra en construcción, a medida que aumentan los casos en el mundo. Se estarán haciendo actualizaciones de esta guía, según se tenga nueva información.

\section{Utilidad de la broncoscopia en pacientes con sospecha o confir- mados de infección por SARS-CoV-2}

- Utilidad limitada: se podría considerar solo en condiciones en las que se vea comprometida la vida del paciente, como hemoptisis o atelectasias masivas, que no responden a otros manejos convencionales, obstrucción de la vía aérea, entre otras; y eventualmente en pacientes con un posible diagnóstico alternativo en el que la broncoscopia deba realizarse con urgencia y en el que el procedimiento permita cambiar rápidamente conductas terapéuticas que beneficien la vida del individuo (7-9).
${ }^{1}$ Medico Especialista en Medicina Interna y Neumología, Miembro del Comité de Broncoscopia de la Asociación Colombiana de Neumología y Cirugía de Tórax

${ }^{2}$ Neumóloga Intervencionista, Centro Médico Imbanaco, Cali, Colombia.

${ }^{3}$ Instituto Neumológico del Oriente, Universidad Industrial de Santander, Bucaramanga, Colombia.

${ }^{4}$ Neumóloga Intervencionista, Hospital Universitario San Ignacio, Pontificia

Universidad Javeriana, Bogotá, Colombia.

${ }^{5}$ Neumólogo Intervencionista, Clínica de Marly, Bogotá, Colombia.

${ }^{6}$ Neumólogo Intervencionista, Unidad de Neumología, CardioVID, Medellín, Colombia.

${ }^{7}$ Neumólogo intervencionista en Oncólogos de Occidente, Armenia, Colombia

${ }^{8}$ Neumóloga Intervencionista, Fundación Clínica Valle del Lili, Cali, Colombia.

${ }^{9}$ Neumólogo en Clínica Fátima, Pasto, Colombia.

${ }^{10}$ Neumólogo Intervencionista, Fundación Neumológica Colombiana; Universidad de la Sabana, Bogotá, Colombia.

${ }^{11}$ Neumólogo NEUMOVIDA S.A.S, Armenia, Colombia.

${ }^{12}$ Neumólogo IPS Universitaria Clínica León XIII, Universidad de Antioquia. Medellín, Colombia.

(Con la participación de la Asociación Colombiana de Broncoscopia y Neumología intervencionista (ACBNI).

Autor de correspondencia: Isabel Palacios-Ortega.

Correo electrónico:

isabel.palacios@imbanaco.com.co

Recibido: 30/04/2020

Aceptado: 16/05/2020 
- Métodos preferidos para recolección de muestras en pacientes con sospecha de infección por SARSCoV-2 $(10,11)$.

- Hisopado nasofaríngeo u orofaríngeo. Se muestra un video ilustrativo en la siguiente dirección https://www.nejm.org/doi/full/10.1056/NEJMvem2010260 (12)

- Aspirado nasofaríngeo

- Aspirado traqueal

- En caso de que un paciente con SARS-CoV-2, confirmado o sospechoso, requiera una broncoscopia por una circunstancia no considerada como emergencia se sugiere esperar mínimo 4 semanas y negativización de la prueba para realizar el procedimiento.

\section{Recomendaciones para broncoscopia en tiempos de pandemia para pacientes sin sospecha de infección por SARS-CoV-2 o en casos de pruebas negativas (6-8)}

\section{Indicación de broncoscopia}

En los pacientes sin infección por SARS-CoV-2 (prueba negativa), o sin sospecha clínica, según las definiciones epidemiológicas, se dan indicaciones para priorizar procedimientos, como se describe en la Tabla 1 (7-9).

\section{Tamizaje para SARS-CoV-2 previo al procedimiento}

- Cuestionario de síntomas por teleconsulta u otro medio no presencial en todos los pacientes ambulatorios, 24 horas antes del procedimiento (9).

- Se requiere realizar prueba RT-PCR de SARSCoV-2/COVID-19 previo a la broncoscopia en los siguientes casos (9):

- Paciente con síntomas respiratorios de menos de 2 semanas de evolución o que cumplan con los criterios epidemiológicos del caso, de acuerdo con la normatividad vigente del Ministerio de Salud de Colombia.

- Paciente hospitalizado en Urgencias, salas generales y cuidados intensivos, con síntomas respiratorios agudos o crónicos, con fiebre con o sin infiltrados pulmonares, preferiblemente debe tener prueba SARS-CoV-2 negativa para ir a procedimiento.

Tabla 1. Priorización de procedimientos durante la pandemia por SARS-CoV-2

\begin{tabular}{|c|c|c|}
\hline Broncoscopia de emergencia & Broncoscopia prioritaria & Broncoscopia no urgente o diferible \\
\hline $\begin{array}{l}\text { Estenosis traqueal moderada a } \\
\text { severa }\end{array}$ & Masa pulmonar sin diagnóstico & Estenosis traqueal leve \\
\hline $\begin{array}{l}\text { Obstrucción bronquial central } \\
\text { severa }\end{array}$ & Masa mediastinal & \\
\hline Hemoptisis masiva & Lavado pulmonar (en proteinosis alveolar) & Sospecha de sarcoidosis \\
\hline Migración de endoprótesis & Extracción de cuerpo extraño & $\begin{array}{l}\text { Enfermedad pulmonar intersticial } \\
\text { crónica }\end{array}$ \\
\hline \multirow{3}{*}{$\begin{array}{l}\text { Atelectasias que comprometan } \\
\text { la oxigenación o la mecánica } \\
\text { ventilatoria }\end{array}$} & Hemoptisis leve a moderada & Tos crónica \\
\hline & $\begin{array}{l}\text { Estudios de infiltrados en inmunosuprimido } \\
\text { Sospecha de tuberculosis, infección } \\
\text { pulmonar no SARS-CoV-2 }\end{array}$ & $\begin{array}{l}\text { Termoplastia bronquial, válvulas } \\
\text { endobronquiales o coils }\end{array}$ \\
\hline & $\begin{array}{l}\text { Ultrasonido bronquial en adenopatías } \\
\text { mediastinales y cáncer pulmonar que } \\
\text { puedan cambiar de estadio }\end{array}$ & \\
\hline
\end{tabular}


- Paciente inmunosuprimido con síntomas respiratorios, con o sin infiltrados pulmonares, preferiblemente debe tener prueba RT-PCR de SARS-CoV-2/COVID-19 negativa para ir a procedimiento.

\section{Consentimiento informado}

Se sugiere advertir al paciente que su procedimiento será llevado a cabo en situación de pandemia y dejarlo por escrito en consentimiento informado. Se propone adjuntar el siguiente párrafo: "He sido informado del estado de pandemia por coronavirus, de que existen incertidumbres sobre el comportamiento clínico de la enfermedad y de la precisión diagnóstica de los test. Se me ha informado que me aplicarán las medidas de protección que establecen los protocolos, pero soy conocedor que no existe un riesgo cero de la transmisión de la enfermedad" $(13,14)$.

\section{Equipo de protección personal (EPP)}

Dado que nos encontramos en fase de mitigación de la enfermedad por COVID-19, y teniendo en cuenta la alta exposición a aerosoles en procedimiento broncoscópico, debe considerarse a todos los pacientes como potencialmente infectados por SARS-CoV-2, y se deben usar los EPP que garanticen al personal de salud la mejor protección. Asimismo, se recomienda limitar el número de personas que asisten al procedimiento (7-9, 14, 15). Algunas medidas son:

- Vestido quirúrgico

- Gorro

- Lavado de manos con agua y jabón, según la recomendación de la Organización Mundial de la Salud (OMS), indiscutible antes y después del procedimiento

- Higienización con alcohol glicerinado durante la aplicación y retiro de cada pieza del EPP

- Traje antifluido completo (ideal) o escafandra o esclavina, según disponibilidad y protocolos de la institución

- Polainas
- Mascarilla N95 para todo el personal de la sala, en todos los casos, sin importar la indicación del procedimiento. También se puede considerar, y más aún en la coyuntura de desabastecimiento de N95, el uso de respiradores o máscaras elastoméricas N100, siguiendo recomendaciones de uso del proveedor de limpieza y desinfección en cada procedimiento

- En ningún caso realizar broncoscopia con mascarilla quirúrgica sencilla

- Monogafas siempre

- Máscara o protector facial siempre

- Higiene de manos

- Primer par de guantes

- Bata quirúrgica antifluido, una bata por paciente

- Segundo par de guantes por encima de las mangas de la bata antifluido

- Se desaconseja el uso de caja de Taiwán debido a la falta de evidencia, estandarización, dificultades en su uso y ausencia de capacitación.

\section{Secuencia de colocación y retiro de acuerdo con la normatividad institucional}

Se sugiere revisar el video ilustrativo de Chestnet.org en https://youtu.be/bG6zISnenPg. En la Tabla 2 se adjunta el orden propuesto de uso del EPP.

\section{Sobre la sala de broncoscopia $(7-9,16)$}

- Limitar el personal de ingreso a la sala

- Preferible una sala con presión negativa o, en su defecto, con adecuado recambio de aire

- Debe haber completa limpieza y desinfección de sala, según protocolo institucional, después de cada paciente

- Recambio de sala, 1 hora

- Se recomienda una valoración por seguridad y salud en el trabajo y del Comité de Prevención de Infecciones y Vigilancia en Salud Pública, mediante una visita a las salas de broncoscopia o de procedimientos. 


\section{Sobre la anestesia (9)}

- Evitar el uso de anestésico local atomizado en orofaringe.

- En lo posible, con sedación o anestesia general, según la indicación de cada paciente.

- Todo paciente con sospecha o SARS-CoV-2 confirmado debe ir bajo anestesia general con tubo orotraqueal.

\section{Después de procedimiento de broncoscopia (9)}

- Recuperación en la sala de presión negativa, en caso de paciente con SARS-CoV-2.
- Dar al paciente máscara facial quirúrgica en todos los casos.

- Si el paciente requiere oxígeno con cánula nasal, usar máscara quirúrgica sobre el rostro del paciente. Si se usa oxígeno con máscara facial, usar sobre la mascarilla quirúrgica.

- Lavado y desinfección de manos con agua y jabón de todo el personal. El uso de guantes no reemplaza el lavado de manos posprocedimiento.

- Desinfección de alto nivel del broncoscopio en todos los casos.

- Limpieza y desinfección de la sala, según el protocolo institucional.

Tabla 2. Propuesta de uso del equipo de protección personal

\begin{tabular}{|c|c|}
\hline Colocación de equipo de protección personal & Retiro del equipo de protección personal \\
\hline 1. Higiene de manos con agua y jabón & 1. Retire el segundo par de guantes \\
\hline 2. Vestido quirúrgico, traje antifluido, gorro o esclavina & $\begin{array}{l}\text { 2. Desanude la bata, retírela tirando del cuerpo hacia } \\
\text { afuera y adelante, enrollándola y deséchela }\end{array}$ \\
\hline 3. Máscara N95 & $\begin{array}{l}\text { 3. Retire el protector facial, tomándolo de atrás, y retire las } \\
\text { monogafas, tomándolas de los tirantes o los laterales. } \\
\text { No toque el frente de la careta o las monogafas. No } \\
\text { toque la parte frontal de la máscara y retírela tirando de } \\
\text { los elásticos }\end{array}$ \\
\hline 4. Monogafas y protección facial & 4. Retire el respirador N95 \\
\hline 5. Primer par de guantes & 5. Retire la esclavina, el traje antifluido y el gorro \\
\hline 6. Bata antifluido & 6. Retire el último par de guantes \\
\hline \multirow[t]{2}{*}{ 7. Segundo par de guantes } & 7. Lavado de manos \\
\hline & $\begin{array}{l}\text { Siempre higienice sus manos con alcohol glicerinado } \\
\text { después del retiro de cada componente de su EPP }\end{array}$ \\
\hline
\end{tabular}

Adaptado de: Chest [Internet]. Disponible en: https://www.chestnet.org/-/media/chesnetorg/Guidelines-and-Resources/Documents/PPE-for-COVID.ashx?la=en\&hash=B4D83DDC4338827DCE2278B072FA4E2DB23919E3 


\section{Conclusión}

La enfermedad por COVID-19 ha generado grandes retos para la comunidad médica. Dado el desconocimiento y la incertidumbre que ha generado con su aparición, se ha hecho necesario generar estudios que nos permitan definir métodos diagnósticos, tratamientos y vacunación. Ahora conocemos el alto índice de contagio entre el personal de salud durante la pandemia, tenemos información sobre su transmisión y el alto riesgo de contagio por procedimientos generadores de aerosoles, entre ellos, los procedimientos endoscópicos. Con el creciente número de personas con infección por SARS-CoV-2, el conocido porcentaje de personas asintomáticas, así como las limitaciones en la realización de pruebas diagnósticas, no queda duda de que las medidas de protección personal para el personal deberán ser estrictas, asumiendo a cada paciente como potencial infectado.

\section{Referencias}

1. World Health Organization [Internet]. Pneumonia of unknown cause - China. Última visita: 14 de febrero de 2020. Disponible en: https:/www.who.int/csr/don/05-january-2020-pneumonia-of-unkown-cause-china/en/

2. World Health Organization [Internet]. Report of the WHO-China joint mission on coronavirus disease 2019 (COVID-2019). Última visita: 24 de febrero de 2020. Disponible en: http:// www.who.int/docs/default-source/coronaviruse/who-chinajoint-mission-on-covid-19-final-report.pdf

3. Kupferschmidt K (Science) [Internet]. Study claiming new coronavirus can be transmitted by people without symptoms was flawed. Última visita: 4 de febrero de 2020. Disponible en: https://www.sciencemag.org/news/2020/02/paper-nonsymptomatic-patient-transmitting-coronavirus-wrong

4. Tran K, Cimon K, Severn M, Pessoa-Silva CL, Conly J. Aerosol generating procedures and risk of transmission of acute respiratory infections to healthcare workers: a systematic review. PLoS One. 2012;7(4):e35797. doi:10.1371/journal. pone. 0035797 .

5. Guan WJ, Ni ZY, Hu Y, Liang W, Ou C, He J, et al. Clinical characteristics of coronavirus disease 2019 in China. N Engl J Med. 2020;382(18):1708-20. doi:10.1056/NEJMoa2002032.

6. van Doremalen N, Bushmaker T, Morris DH, Gamble A, Williamson BN, Tamin A, et al. Aerosol and surface stability of SARS-CoV-2 as compared with SARS-CoV-1. N Engl J Med. 2020;382:1564-7. doi: 10.1056/NEJMc2004973.
7. Wahid MM, Lamb C, Murgu S, Musani A, Shojaee S, Sachdeva A, et al. American Association for Bronchology and Interventional Pulmonology (AABIP) statement on the use of bronchoscopy and respiratory specimen collection in patients with suspected or confirmed COVID-19 infection. Disponible en: https://aabronchology.org/wp-content/uploads/2020/03/2020AABIP-Statement-on-Bronchoscopy-COVID.GAE-updatedVersion.pdf

8. Group of Interventional Respiratory Medicine, Chinese Thoracic Society. [Expert consensus for bronchoscopy during the epidemic of 2019 novel coronavirus infection (Trial version)]. Zhonghua Jie He He Hu Xi Za Zhi. 2020;43(3):199-202. doi: 10.3760/cma.j.issn.1001-0939.2020.03.012.

9. Wahidi MM, Shojaee S, Lamb CR, Ost D, Maldonado F, Eapen $\mathrm{G}$, et al. The use of bronchoscopy during the COVID-19 pandemic: CHEST/AABIP Guideline and Expert Panel Report. Chest. 2020;158(3):1268-81. doi: 10.1016/j. chest.2020.04.036.

10. Grupo ACIN-IETS del Consenso Colombiano para Recomendaciones de Atención COVID-19. Consenso colombiano de atención, diagnóstico y manejo de la infección por SARSCoV-2/COVID-19 en establecimientos de atención de la salud. Rev Colomb Infectología. 2020;24(3):1-6.

11. Ministerio de Salud y Protección Social. Lineamientos para el uso de pruebas diagnósticas de SARS-COV-2 (COVID-19) en Colombia. 2020.

12. Marty FM, Chen K, Verrill KA. How to obtain a nasopharyngeal swab. N Engl J Med. 2020;10.1056/NEJMvcm2010260. doi:10.1056/NEJMvem201026.

13. Crespo J, Andrade R, Alberca de Las Parras F, Balaguer F, Barreiro-de Acosta M, Bujanda L, et al. Resumption of activity in gastroenterology departments. Recommendations by SEPD, AEEH, GETECCU and AEG. Gastroenterol Hepatol. 2020;43(6):332-47. doi: 10.1016/j.gastrohep.2020.04.001.

14. Verbeek JH, Rajamaki B, Ijaz S, Sauni R, Toomey E, Blackwood B, et al. Personal protective equipment for preventing highly infectious diseases due to exposure to contaminated body fluids in healthcare staff. Cochrane Database Syst Rev. 2020;4:CD011621. doi: 10.1002/14651858.CD011621.pub4.

15. Feldman O, Meir M, Shavit D, Idelman R, Shavit I. Exposure to a surrogate measure of contamination from simulated patients by emergency department personnel wearing personal protective equipment. JAMA. 2020;323(20):2091-3. doi:10.1001/jama.2020.6633.

16. Repici A, Maselli R, Colombo M, Gabbiadini R, Spadaccini M, Anderloni A, et al. Coronavirus (COVID-19) outbreak: what the department of endoscopy should know. Gastrointest Endosc. 2020;92(1):192-7. doi: 10.1016/j.gie.2020.03.019.espec 